\title{
Pituicytoma: Report of two cases
}

\author{
ZHIGANG MAO ${ }^{1}$, WEIWEI XIAO ${ }^{2}$, HAIJUN WANG ${ }^{1}, \mathrm{ZHI} \mathrm{LI}^{3}$, \\ QUAN HUANG $^{1}$, DONGSHENG HE ${ }^{1}$ and YONGHONG ZHU ${ }^{2}$ \\ ${ }^{1}$ Department of Neurosurgery, the First Affiliated Hospital; ${ }^{2}$ Department of Histology and Embryology, \\ Zhongshan School of Medicine; ${ }^{3}$ Department of Pathology, the First Affiliated Hospital, \\ Sun Yat-sen University, Guangzhou, Guangdong 510080, P.R. China
}

Received April 20, 2010; Accepted October 14, 2010

DOI: 10.3892/ol.2010.209

\begin{abstract}
This report describes two cases of pituicytoma. The two patients were female and presented with visual complaints, diabetes insipidus, headaches and menstrual disorders. The imaging characteristic was an intrasellar or suprasellar mass, and one mass originated in the pituitary stalk. The mass showed homogeneous enhancement with contrast administration. A hematoxylin and eosin stain showed a compact structure consisting of elongated, bipolar spindle cells arranged in interlacing fascicles or assuming a storiform pattern. Immunohistochemically, the tumor showed diffuse strong expression of S-100 protein, vimentin and epithelial membrane antigen, and glial fibrillary acidic protein was focally positive. The pituicytoma may have originated in the pituitary stalk and presented with diabetes insipidus, and the differential diagnosis should be compared with the pituitary stalk mass. Bleeding during resection was moderate, and surgery was regarded as the first choice of treatment for pituicytomas.
\end{abstract}

\section{Introduction}

Pituicytoma is a rare benign neoplasm occurring in the sellar and suprasellar regions and it originates in the neurohypophysis or infundibulum. Scothorne reported the first case in 1955 (1). Since then, 28 additional cases (not including the present two cases) of this neoplasm have been reported (1-14), including one autopsy case (5). It is difficult to identify pituicytomas from other sellar and suprasellar neoplasms, such as granular

Correspondence to: Dr Yonghong Zhu, Department of Histology and Embryology, Zhongshan School of Medicine, Sun Yat-sen University, No. 74 Zhongshan 2nd Road, Guangzhou, Guangdong 510080, P.R. China

E-mail: zhuyongh@mail.sysu.edu.cn

Dr Haijun Wang, Department of Neurosurgery, the First Affiliated Hospital, Sun Yat-sen University, No. 58 Zhongshan 2nd Road, Guangzhou, Guangdong 510080, P.R. China

E-mail: wzwxr_1995@yahoo.com.cn

Key words: pituicytoma, neurohypophysis, pituitary stalk, granular cell tumor cell tumors and pilocytic astrocytomas. The diagnosis of the neoplasms was based on a pathological examination. The neoplasms were found to be histologically benign, but their hypervascular nature makes surgical resection difficult (2). Local recurrence following subtotal resection is common (3). The clinical and surgical features of two cases of pituicytomas and the relevant literature is reviewed and evaluated.

\section{Case report}

Case one. A 47-year-old female without prior medical history described a six-month history of headaches and menstrual disorder. Visual function was normal, and preoperative neuroendocrine studies were normal apart from a slightly elevated prolactin level of $45 \mathrm{ng} / \mathrm{ml}$, thought to be secondary to the pituitary stalk disinhibition effect. Magnetic resonance imaging (MRI) scans showed a 16x13x10 mm solid mass. The mass exhibited intermediate signal intensity on T1-weighted images, intermediate, slightly increased signal intensity on T2-weighted images, and marked homogeneous enhancement with contrast administration, and the neurohypophysis signal had disappeared. It appeared to be centered on the posterior lobe. The computed tomography (CT) images did not reveal calcifications, necrosis, bone destruction or hyperostosis.

A transsphenoidal microsurgical approach was selected for tumor resection. A soft, reddish, minor vascular mass was found in the neurohypophysis. A total resection was performed, in order topreserve the pituitary stalk, and normal hypophysis was observed during the resection. The final pathological diagnosis was pituicytoma. No adjuvant treatment was administered. No residual or regrowth of the tumor was noted, as shown by MRI scans, over a follow-up period of 18 months (Fig. 1).

Case two. A 51-year-old female presented with one year of visual complaints, six months of diabetes insipidus and two months of headaches. Visual field testing showed lefttemporal hemianopsia. The results of preoperative neuroendocrine studies were normal apart from an elevated prolactin level of $188 \mathrm{ng} / \mathrm{ml}$. The MRI scan showed a suprasellar and post-chiasmatic mass, and the tumor measured 9x7x6 mm. The mass exhibited intermediate signal intensity on T1-weighted images, intermediate, slightly increased signal intensity on 

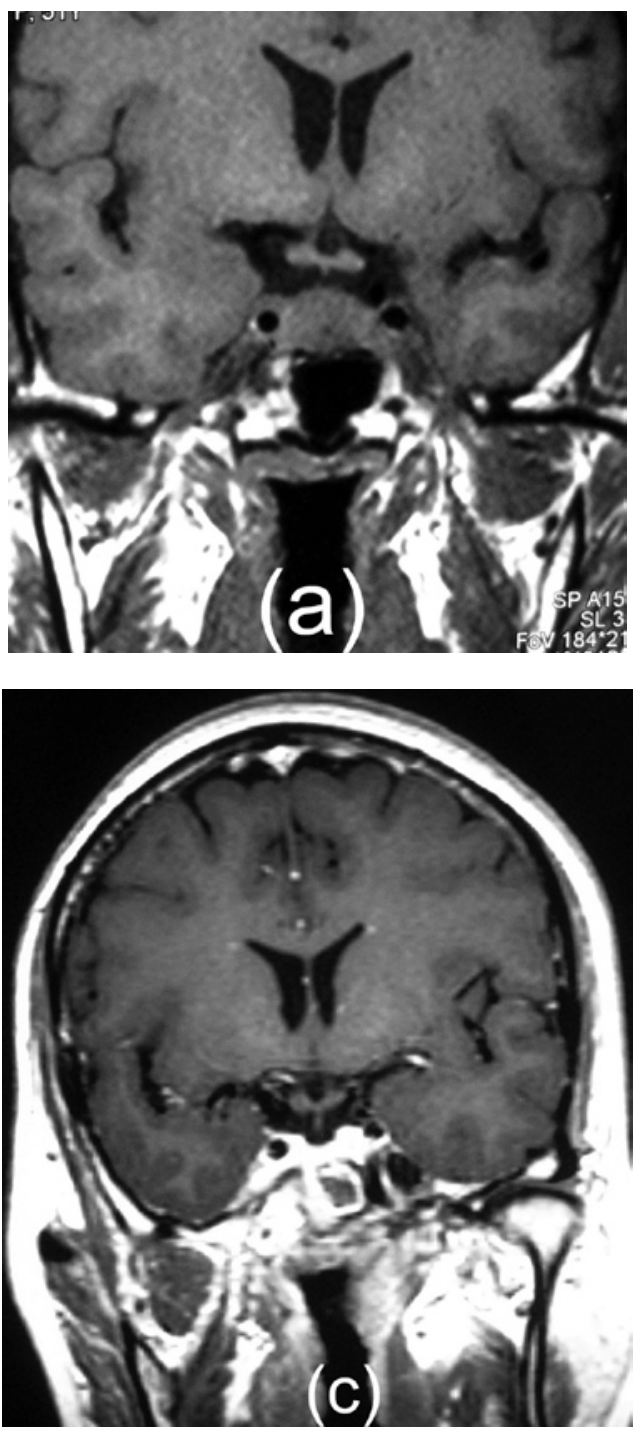
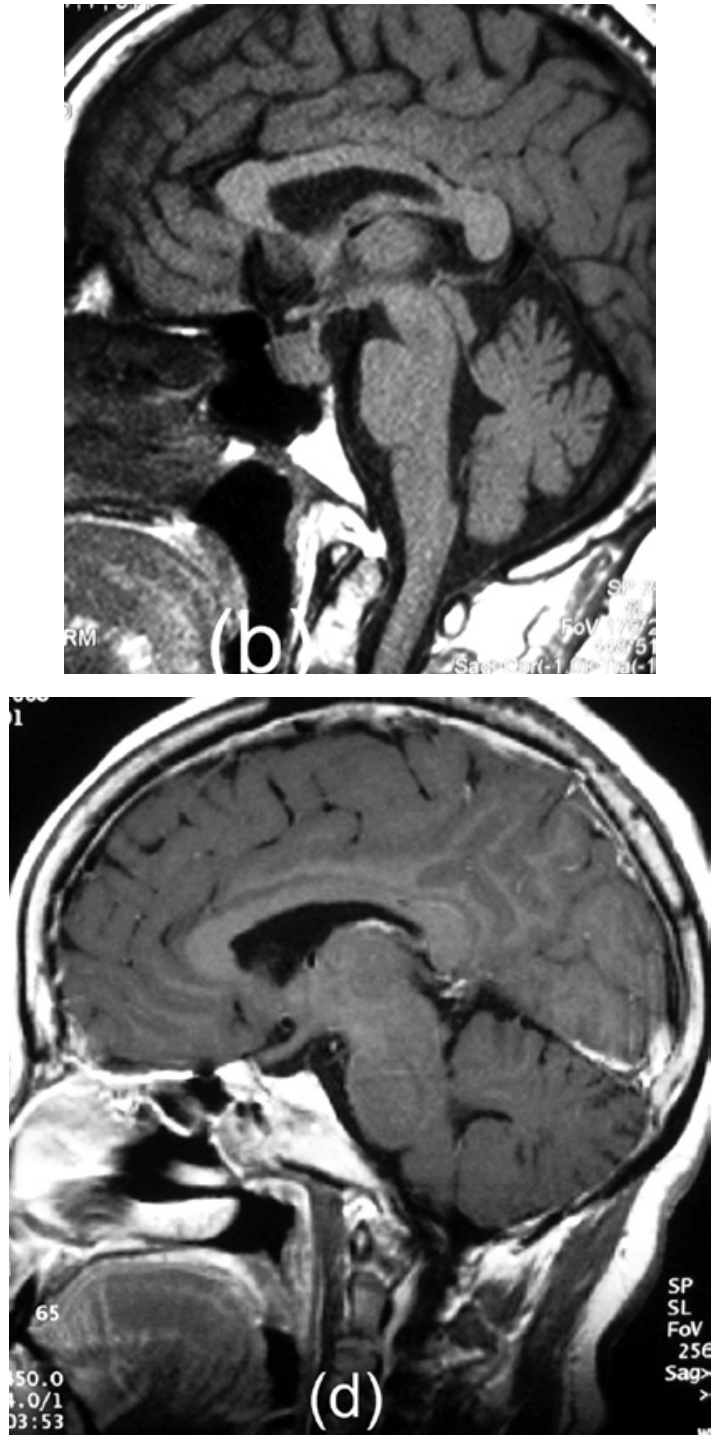

Figure 1. (a and b) T1W1-weighted coronal and sagittal MRI scans showing the intersellar mass. (c and d) T1-weighted contrast-enhanced coronal and sagittal MRI scans for the same patient showed no regrowth or residual mass 18 months post-operatively.

T2-weighted images, and marked homogeneous enhancement with contrast administration. The CT images showed no calcifications, necrosis, bone destruction or hyperostosis. The mass was attached to the pituitary stalk and infundibulum, and appeared to have originated from the pituitary stalk, causing compression of the left optic chiasm.

The patient underwent a right pterional craniotomy resection. A soft, reddish, moderately vascular mass was adherent to the pituitary stalk. A total resection was performed, to preserve the pituitary stalk. The visual acuity and visual field defects improved after surgery. The diabetes insipidus was moderated after surgery, and desmopressin was prescribed at a dose of $0.1 \mathrm{mg} / \mathrm{day}$. The patient was discharged on a low dose of dexamethasone. Endocrinological studies after discharge revealed panhypopituitarism requiring thyroid and adrenal hormone replacement. A follow-up MRI scan obtained with gadolinium showed no residual mass 11 months after the surgery (Fig. 2).

Pathological findings. A hematoxylin and eosin (H\&E) stain showed that the tumor was predominantly a compact structure consisting of elongated, bipolar spindle cells arranged in interlacing fascicles or assuming a storiform pattern (Fig. 3). The cytoplasm was lightly eosinophilic. No significant cellular or nuclear pleomorphism, mitotic activity, necrosis, cytoplasmic granules, eosinophilic granular bodies or evidence of brain invasion were observed.

Immunohistochemical stain was performed on formalinfixed paraffin-embedded tissue using the Envision system (DakoCytomation, Carpinteria, CA, USA). Antigen retrieval was performed by utilizing steam heat. Primary antibodies against the following antigens were used: S-100 protein (polyclonal, ready-to-use, Code no. IR504, Dako), vimentin (monoclonal, ready-to-use, N-Series primary antibody, Code no. 1583, Dako), epithelial membrane antigen (EMA, monoclonal, ready-to-use, Dako), glial fibrillary acidic protein (GFAP, polyclonal, 1:500, Dako), Ki-67 (monoclonal MIB-1, 1:50, Code no. M7248, Dako), neuron-specific enolase (NSE, Code no. IR612, ready-to-use, Dako), neurofilament (monoclonal, Code no. IR607, ready-to-use, Dako ), chromogranin A (polyclonal, Code no. A0430, 1:500, Dako), prolactin (polyclonal, Code no. A0569, 1:200, Dako), catabolite 

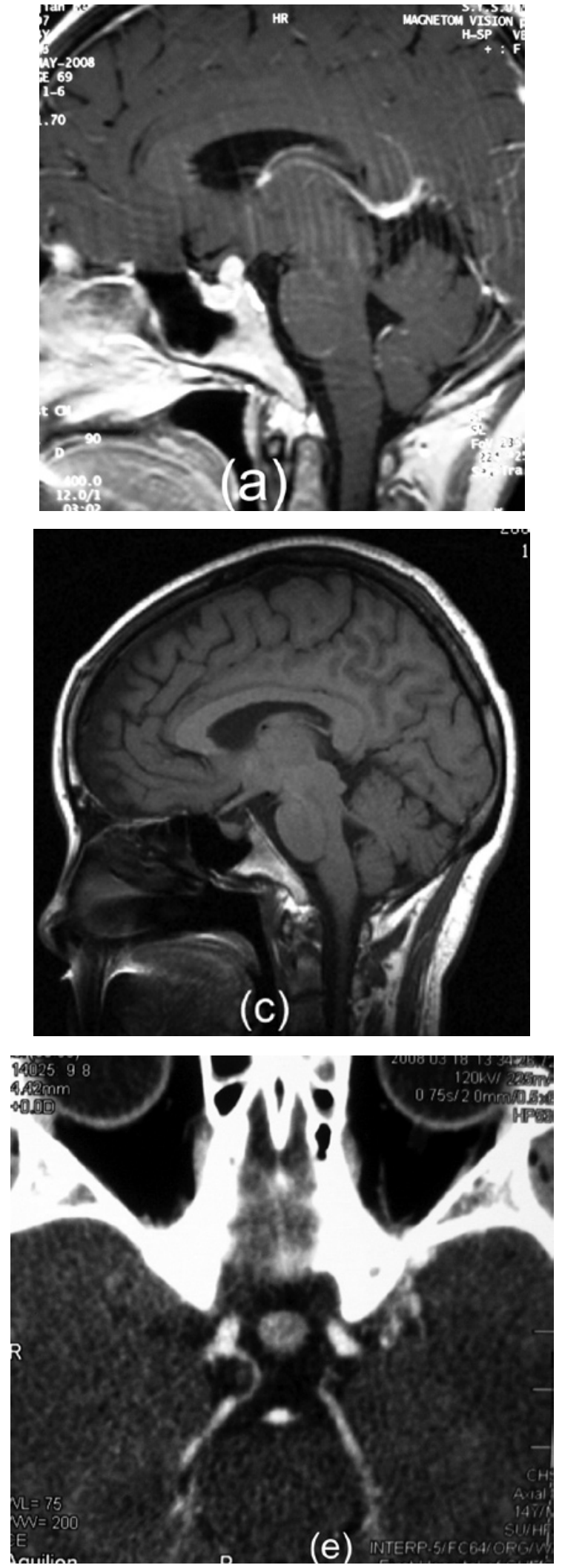

gene activator (CgA) (polyclonal, Code no. A0569, 1:200, Dako), growth hormone (polyclonal, Code no. A0570, 1:400, Dako), follicle stimulating hormone (monoclonal, Code no. M3504, 1:50, Dako), luteinizing hormone (monoclonal, Code no. M3502, 1:50, Dako), thyroid stimulating hormone (monoclonal, Code no. M3503, 1:50, Dako) and adrenocorticotropin (monoclonal, Code no. M3501, 1:50, Dako). The tumor showed
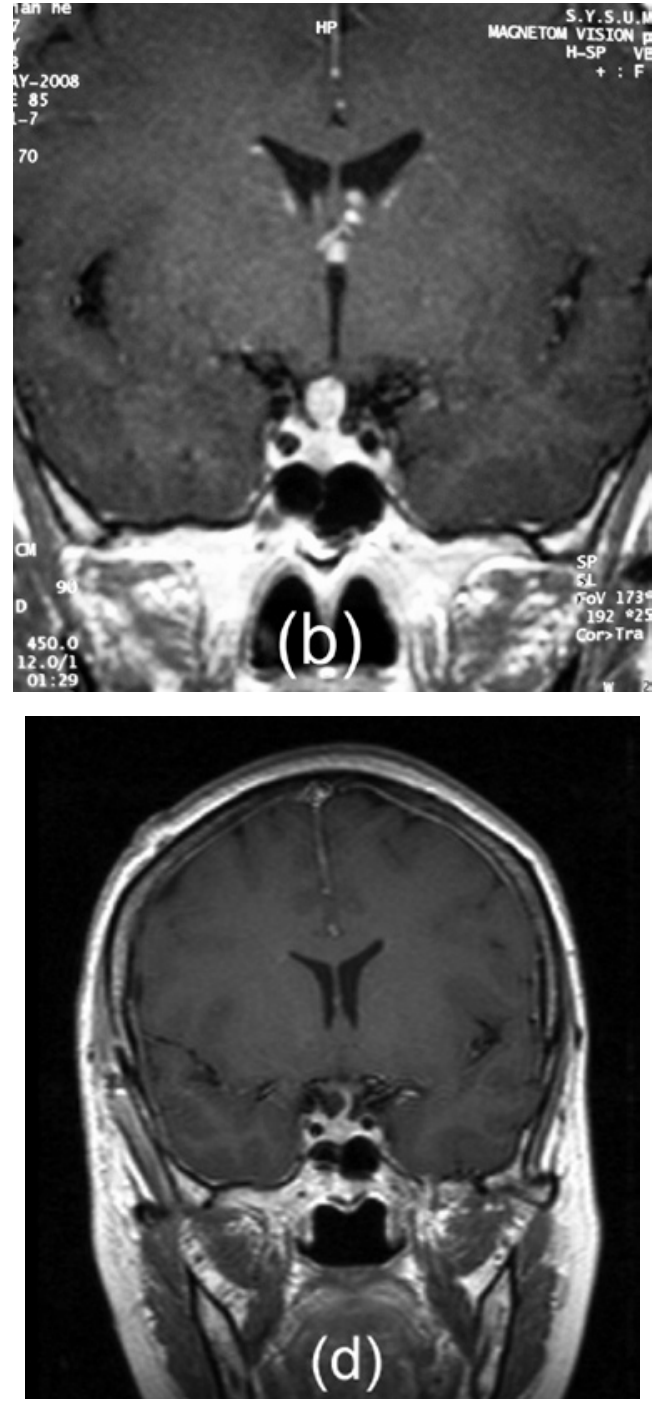

Figure 2. (a and b) T1W1-weighted contrast-enhanced sagittal and coronal MRI scans showing the heterogeneous enhancement of the suprasellar mass, which appears to have originated from the suprasellar region with well-defined margins and tumor causing compression of the chiasm. The pituitary stalk is not regarded as a structure separate from the mass. (c and d) T1-weighted contrast-enhanced sagittal and coronal MRI scans for the same patient showed no regrowth or residual mass adherent to the pituitary stalk 11 months post-operatively. (e) CT contrast-enhanced axial scans showing the heterogeneous enhancement of the lesion prior to surgery.

diffuse strong immunoreactivity of S-100 protein, vimentin and EMA, and GFAP showed a focal, weak positive reaction. Negative reactions were noted for NSE, neurofilament, chromogranin A, CgA and synaptophysin. Ki-67 (MIB-1) labeling was $<1 \%$. The anterior pituitary hormone was also negative.

\section{Discussion}

A total of 1,490 consecutive patients underwent transsphenoidal resections of the pituitary adenomas in our hospital between January 1996 and December 2008. Approximately half of the adenomas were macroadenomas (15). Among these, we identified two pituicytomas. A total of 30 bona fide examples have been described thus far, often as case reports. Pituicytomas are rare and little is known about the epidemiology (4). There 

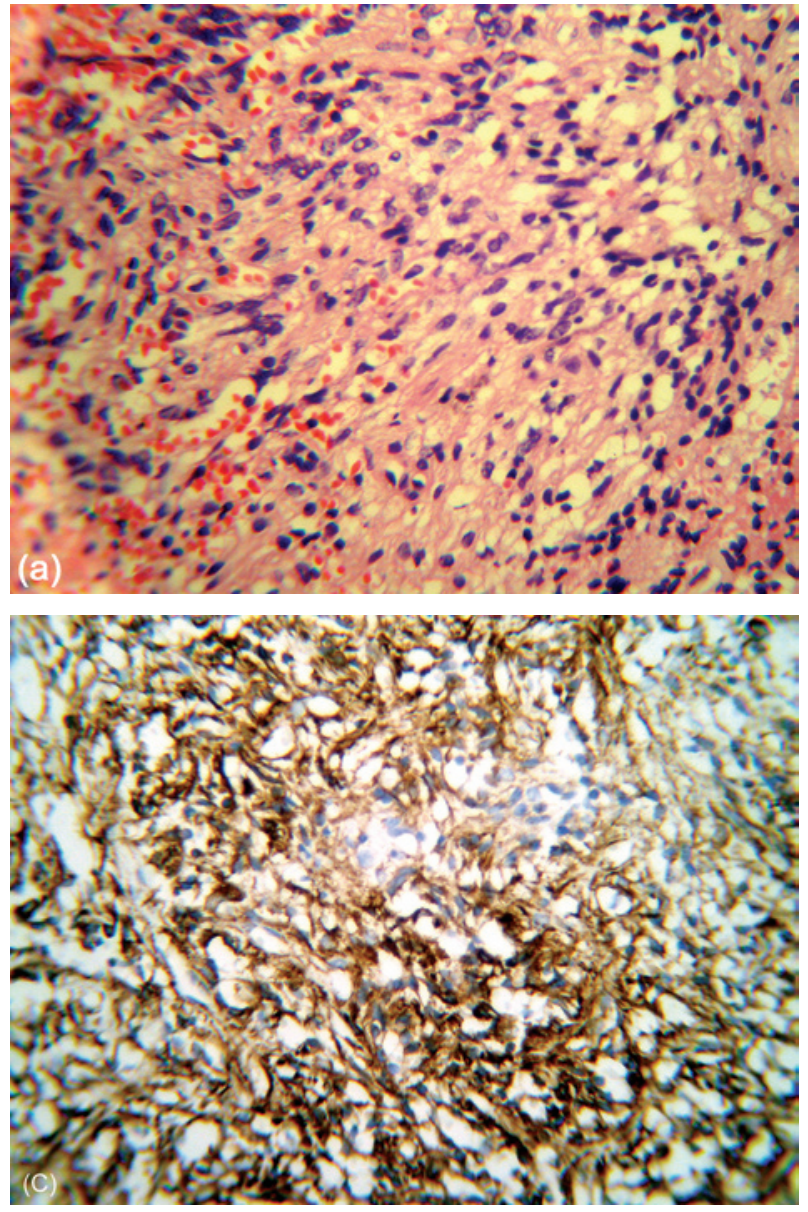
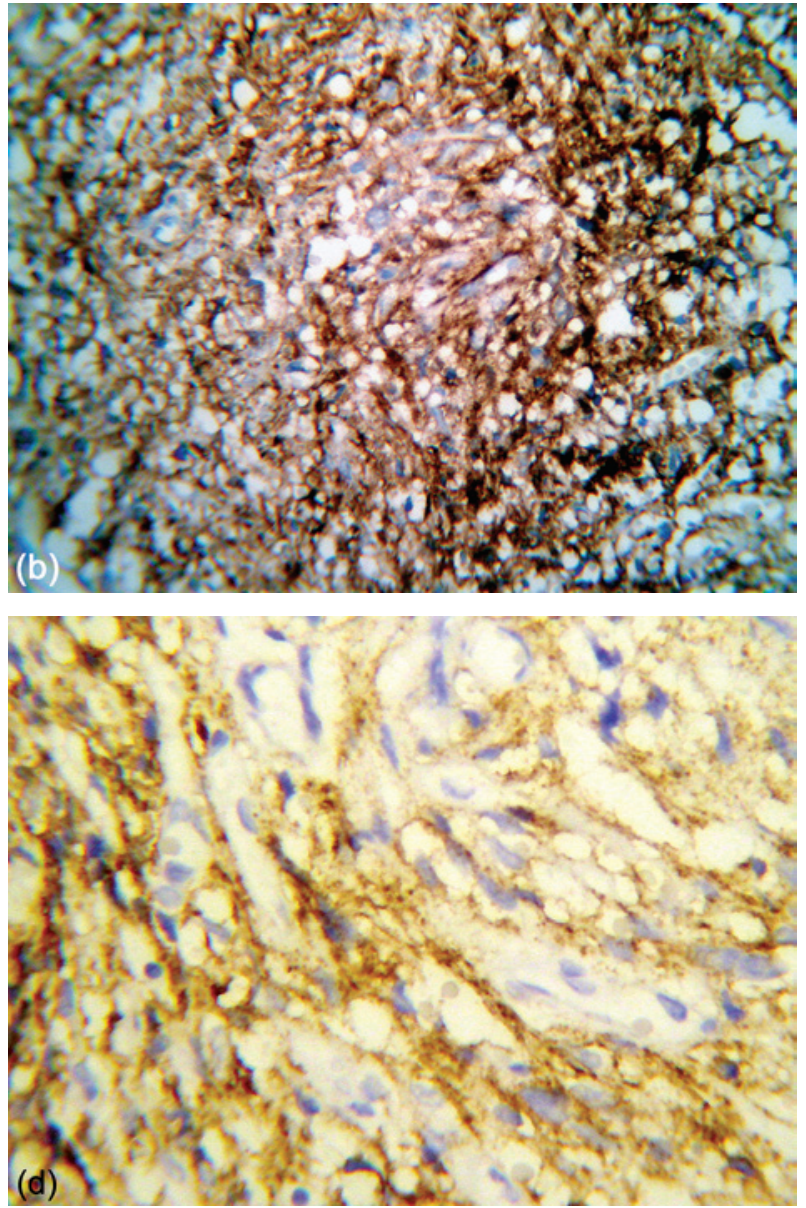

Figure 3. (a) Microphotographs of the H\&E sections show bipolar spindle cells with no pleomorphism and mitotic activity; original magnification, $x$ 100. (b) Immunohistochemically, the tumor shows strong and diffuse staining for S-100 in the cytoplasm and nuclei of the tumor cells; original magnification, $x 100$. (c) Immunohistochemically, cytoplasmic and focal nuclear immunoreactivity for vimentin is shown; original magnification, $x$ 100. (d) Immunohistochemically, the tumor shows strong and diffuse staining for epithelial membrane antigen; original magnification, $\mathrm{x} 200$.

appears to be a slight predominance in males $(14,16)$. Since the cell of origin remained undetermined, nosological confusion surrounded the use of the term pituicytoma, which is also termed infundibuloma, granular cell myoblastoma, choristoma and granular cell tumor (16). Pituicytoma is now included and described as an indolent World Health Organization grade 1 tumor that involves the posterior pituitary and/or its stalk, is composed of spindle cells, and is derived from pituicytes (17). Pituicytomas appear to be benign, slow-growing tumors presenting as the result of symptoms from mass effect.

As previously reported (1-13), 12 of the 30 patients (40\%) presented with decreased visual acuity or visual field defects, caused by compression of the optic nerves and/or chiasm. A total of 16 of the 30 patients $(53 \%)$ presented with signs of pituitary insufficiency, with the most common endocrinopathy being decreased libido, amenorrhea, hypopituitarism and hypogonadism. A total of 5 patients presented with both visual and endocrinopathic symptoms. A total of 7 of the 30 patients (23\%) presented with headaches. A number of patients also had vague complaints of memory changes, dizziness and fatigue. Only 1 patient presented with tumor apoplexy (6). Of our cases, only 1 patient presented with diabetes insipidus, which was uncommon. These clinical presentations are in concordance with a sellar or suprasellar area mass.
The CT findings are not well reported, as the CT contrastenhanced axial scans revealed heterogeneous enhancement in 1 patient (Fig. 2). The majority of the reported MRI findings of pituicytomas are isointense on T1-weighted images and hyperintense on T2-weighted images, with homogeneous enhancement following the administration of gadolinium $(4,12)$. This presentation was different from the inhomogeneity of granular cell tumors (4). The rapidity of enhancement correlates with the increased vascularity of the lesions, as reported by Gibbs et al (11), Wolfe et al (4) and Uesaka et al (9), and the angiogram revealed a significant vascular blush $(4,9)$.

As previously described (4), the characteristic features of pituicytomas that distinguish them from pilocytic astrocytomas or schwannomas include strongly positive staining for S-100 and vimentin, and focally positive staining for GFAP. EMA showed a strong expression in the 2 patients. The transmembrane glycoprotein of EMA is encoded by the MUC1 gene, located on chromosome 1 in the 1q21-24 region. EMA is present in numerous epithelial cells. EMA, an integral mucin complex, is representative of the antigens involved in the secretory processes of glandular epithelia, which change quantitatively during specialization, differentiation and neoplastic transformation (18). However, the role of EMA in pituicytomas requires further study. GFAP is a $54 \mathrm{kDa}$, type III 
intermediate filament protein that is the major constituent of glial filaments in astrocytes (19). GFAP is considered to be a general marker for astrocytes in the central nervous system (20). The accurate diagnosis of brain invasion is therefore critical and, in borderline cases, an immunohistochemical stain for GFAP aids in the delineation of entrapped glial elements within the substance of a brain-invasive meningioma (21).

Our cases are noteworthy for various reasons. Firstly, they add another possible entity to the differential diagnosis of a pituitary stalk and diabetes insipidus mass. The tumor originated in the pituitary stalk in one of our patients. Notably, fewer symptoms of diabetes insipidus were found at presentation than anticipated and were clearly reported in only one case (7). Pituitary stalk lesions are often identified to investigate symptoms such as diabetes insipidus. As noted in the literature, patients who develop pathology involving the stalk commonly present with varying degrees of hypopituitarism, diabetes insipidus and hyperprolactinemia (22).

On the other hand, the two tumors were not highly vascular, and the patients exhibited moderate bleeding from the tumors, which were completely resected during the surgery. Another reason that the two tumors may have been much easier to excise without significant bleeding was due to their smaller size. These findings are not in agreement with some authors. Ulm et al reported that pituicytomas generally were highly vascular, and significant bleeding is often encountered during resection (3,7-10). These authors correctly call attention to the fact that surgery may be difficult due to the location and marked vascularity of the tumors. Heavy intraoperative bleeding hindered a complete resection in certain patients.

No adjuvant treatment was administered to the 2 patients. Follow-up MRI scanning obtained with gadolinium showed no residual or regrowth mass 18 and 11 months post-operatively. Recurrence following a subtotal resection is common, occurring in 4 of the 7 cases reported in the literature for which a follow-up of greater than six months was available $(2,3,7-9)$. With total resection, the prognosis is favorable. None of the patients receiving total resection had recurrences at the reported follow-ups. The highly vascular nature of the tumor and its potential for infiltration can make total resection difficult. Subtotal resection due to extensive bleeding occurred in the two cases reported by Ulm et al (3). A total of 3 patients with subtotal resection underwent postoperative radiation, including 2 with radiosurgery and 1 with fractionated radiation. The role of radiation therapy is uncertain in this rare lesion. Since few cases of pituicytoma have been treated with radiation, and given that the natural history appears similar to that of granular cell tumor, the data available currently should be utilized. The authors are in agreement with Brat et al, who noted that completeness of excision was the most significant predictor of recurrence (2), and that surgery is the first choice of treatment for patients with a pituicytoma.

\section{References}

1. Scothorne CM: A glioma of the posterior lobe of the pituitary gland. J Pathol Bacteriol 69: 109-112, 1955.

2. Brat DJ, Scheithauer BW, Staugaitis SM, Holtzman RN, Morgello S and Burger PC: Pituicytoma: a distinctive lowgrade glioma of the neurohypophysis. Am J Surg Pathol 24: 362-368, 2000 .

3. Ulm AJ, Yachnis AT, Brat DJ and Rhoton AL Jr: Pituicytoma: reports of two cases and clues regarding histogenesis. Neurosurgery 54: 753-757, 2004.

4. Wolfe SQ, Bruce J and Morcos JJ: Pituicytoma: case report. Neurosurgery 63: E173-E174, 2008.

5. Takei H, Goodman JC, Tanaka S, Bhattacharjee MB, Bahrami A and Powell SZ: Pituicy toma incidentally found at autopsy. Pathol Int 55: 745-749, 2005.

6. Benveniste RJ, Purohit D and Byun H: Pituicytoma presenting with spontaneous hemorrhage. Pituitary 9: 53-58, 2006.

7. Figarella-branger D, Dufour H, Femandez C, Bouvier-Labit C, Grisoli F and Pellissier JF: Pituicytomas, a mis-diagnosed benign tumor of the neurohypophysis: report of three cases. Acta Neuropathol 104: 313-319, 2002.

8. Kowalski RJ, Prayson RA and Mayberg MR: Pituicytoma. Ann Diagn Pathol 8: 290-294, 2004.

9. Uesaka T, Miyazono M, Nishio S and Iwaki T: Astrocytoma of the pituitary gland (pituicytoma): case report. Neuroradiology 44: 123-125, 2002.

10. Katsuta T, Inoue T, Nakagaki H, Takeshita M, Morimoto K and Iwaki T: Distinctions between pituicytoma and ordinary pilocytic astrocytoma. J Neurosurg 98: 404-406, 2003.

11. Gibbs WN, Monukie S, Linskey ME and Hasso AN: Pituicytoma: diagnostic features on selective carotid angiography and MR imaging. Am J Neuroradiol 27: 1639-1642, 2006.

12. Thiryayi WA, Gnanalingham KK, Reid H, Heald A and Kearney T: Pituicytoma: a misdiagnosed benign tumour of the posterior pituitary. Br J Neurosurg 21: 47-48, 2007.

13. Nakasu Y, Nakasu S, Saito A, Horiguchi S and Kameya T: Pituicytoma: two case reports. Neurol Med Chir (Tokyo) 46: 152-156, 2006.

14. Shah B, Lipper MH, Laws ER, Lopes B and Spellman MJ: Posterior pituitary astrocytoma: a rare tumor of the neurohypophysis: a case report. Am J Neuroradiol 26: 1858-1861, 2005.

15. ZL Han, DS He, ZG Mao and HJ Wang: Cerebrospinal fluid rhinorrhea following trans-sphenoidal pituitary macroadenoma surgery: experience from 592 patients. Clin Neurol Neurosurg 110: 570-579, 2008

16. Rossi ML, Bevan JS, Esiri MM, Hughes JT and Adams CB: Pituicytoma (pilocytic astrocytoma). Case report. J Neurosurg 67: 768-772, 1987.

17. Rousseaua A, Mokhtaria K and Duyckaertsa C: The 2007 WHO classification of tumors of the central nervous system - what has changed? Curr Opin Neurol 21: 720-727, 2008.

18. Shashikant C, Talal AS and Georges D: Epithelial membrane antigen in hematolymphoid neoplasms: a review. Appl Immunohistochem 5: 203-215, 1997.

19. Eng LF, Ghirnikar RS and Lee YL: Glial fibrillary acidic protein: GFAP-thirty-one-years (1969-2000). Neurochem Res 25: 1439-1451, 2000.

20. Huang QL, Zhao SF, Gaudin A, Quennedey B and Gascuel J: Glial fibrillary acidic protein and vimentin expression in the frog olfactory system during metamorphosis. Neuro Report 16: $1439-1442,2005$

21. Trembath D, Miller CR and Perry A: Gray zones in brain tumor classification evolving concepts. Adv Anat Pathol 15: 287-297, 2008.

22. Rupp D and Molitch M: Pituitary stalk lesions. Curr Opin Endocrinol Diabetes Obes 15: 339-345, 2008. 